\title{
An Assessment of Visual Discomfort Caused by Motion-in-Depth in Stereoscopic 3D Video
}

\author{
Sang-Hyun $\mathrm{Cho}^{1}$ \\ cshgreat@catholic.ac.kr \\ Hang-Bong Kang ${ }^{2}$ \\ hbkang@catholic.ac.kr
}

${ }^{1}$ Dept. of Computer Engineering, The Catholic University of Korea, Gyeonggi-do, Korea

${ }^{2}$ Dept. of Digital Media, The Catholic University of Korea, Gyeonggi-do, Korea

\begin{abstract}
The motion-in-depth is an important factor in measuring visual discomfort when watching stereoscopic 3D video since it changes accommodation and vergence at the same time. In this paper, we examine visual discomfort caused by motion-in-depth in terms of viewing time and display size in watching stereoscopic 3D video. The assessment methods for visual discomfort we used were a subjective test such as a questionnaire, and an objective test such as eye blink rate detection. The experimental results showed that visual discomfort increased in proportion with the speed of motion-in-depth and viewing time. We also found that the viewer's discomfort increased when watching stereoscopic 3D video on the smaller-sized display than on a larger-sized display.
\end{abstract}

\section{Introduction}

Stereoscopic image viewing comfort is one of the main problems that should be solved before the mass market proliferation of stereoscopic 3D content services. The complaints of some viewers about visual discomfort such as eyestrain and associated symptoms including nausea and headaches present a barrier in the popularization of stereoscopic 3D technology. Some visual discomfort may occur due to excessive demand on the accommodation-vergence linkage, fast local and global motion, and various stereoscopic distortions $[1,2,3,4,5,6]$.

Among those factors, we focus here on some basic components in watching stereoscopic 3D content--motion, binocular disparity, viewing time and display size--while analyzing and defining the causes of visual discomfort based on viewers' personal characteristics. Usually, motion in stereoscopic 3D displays combines lateral motion and motion-in-depth.

Recent research suggests that motion-in-depth could play a more important role in generating visual discomfort than lateral motion on vertical and horizontal axes in 
stereoscopic 3D displays [7,8,9]. However, previous studies did not consider other factors like viewing time and display size in evaluating visual discomfort.

The visual discomfort induced by excessive binocular disparity over the fusional range has been studied in $[10,11]$. However, display size should also be considered in evaluating visual discomfort because the perceived depth of stereoscopic 3D content is strongly linked to the size of the display. In [12], the effect of display size is tested among different display sizes. Viewing time is also strongly related to visual discomfort in watching stereoscopic 3D content.

The main contribution of this paper is two-fold: (1) We analyze the effects of motionin- depth, viewing time and display size in measuring visual discomfort. (2) The evaluation method for visual discomfort is proposed by integrating a subjective test such as a questionnaire, and an objective test such as eye blink rate detection.

The rest of this paper is organized as follows. In Section II, we describe motion-indepth perception in stereoscopic 3D video. In Section III, we present our experimental setup, test video specifications, and test procedure. In Section IV, we discuss our experimental results.

\section{Motion-in-depth Perception in Stereoscopic 3D Video}

Object motion in stereoscopic 3D video can be classified into lateral-motion and motion-in-depth. Lateral-motion is perceived in one or two-dimensional space. On the other hand, motion-in-depth perception cannot be fully explained without considering changes in disparity between correlated images. For lateral-motion, both eyes make the same conjunctive eye movements, called version. To perceive motion-in-depth, the eyes make opposite, disjunctive, eye movements, called vergence [13]. On the other hands, accommodation is alteration of the lens to focus the area of interest on the fovea [1].

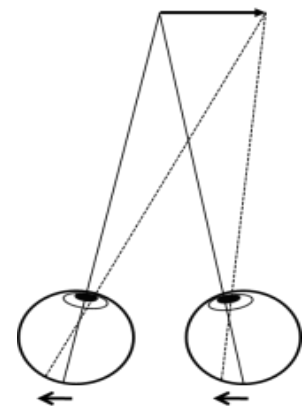

(a)

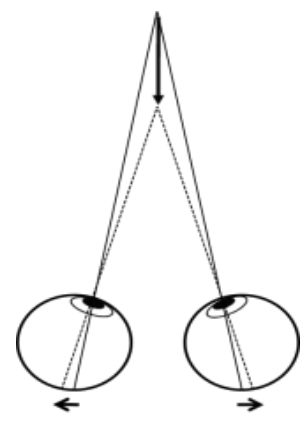

(b)

Figure 1. Object motion in stereoscopic 3D video: (a) lateral; (b) motion-in-depth.

Generally, motion-in-depth induces changes in binocular disparity, which derives the temporally changing demand of accommodation-vergence(AC) linkage. It was reported that the perceptual impact of excessive demand on the AC linkage is related to visual discomfort [1,9]. Yano et al.[14] concluded that the visual fatigue occurred when the 
stereoscopic images involved motion-in-depth even if they were displayed within the range of depth of field. Meanwhile, no visual fatigue was found in lateral-motion images. Recently, Jung et al.[9] examined the relation between visual comfort and object motion characteristics in stereoscopic 3D videos. Lambooij et al.[1] addressed that, even within a disparity range of comfortable viewing, visual discomfort might still occur due to an excessive demand on the AC linkage caused by fast motion-in-depth. Therefore, it is necessary to investigate the relationship between visual discomfort and motion-in-depth with respect to diverse speed, viewing time and display sizes.

\section{Experimental Design}

\subsection{Experimental Setup}

The design of the experimental environment was in line of the recommendations of ITU-R BT.500-13 [15]. The experimental setup is shown in Figure 2 with the following specifications:

- Size: 55inch (passive type), 27inch (passive type)

- Aspect ratio: $16: 9$

- Spatial Resolution: $1920 * 1080$

- Environmental luminance on the screen: 200 lux

- Participants: 20 subjects (14 males and 6 females, ages 20 35: medical condition checked)

Lighting conditions were held constant for all participants during all sessions. Any external illumination was completely blocked out by thick curtains. The temperature and humidity were maintained constantly and there were no vibrations or strong odors.

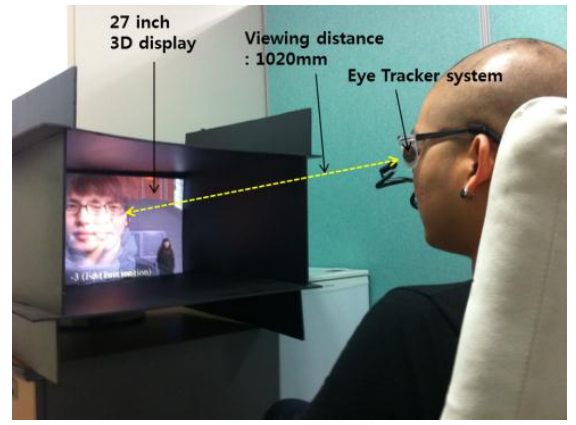

(a)

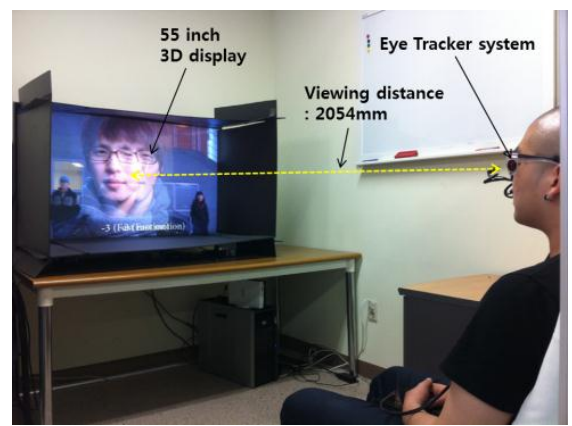

(b)

Figure 2. Experimental setup : (a) 27 inch 3D TV; (b) 55 inch 3D TV.

\subsection{Test Videos}

Because there are no publicly available stereoscopic 3D content suitable for our purpose of measuring the viewer's visual discomfort, we produced stereoscopic 3D video featuring diverse parallax angle variations and a salient object's motions. Each video clip contained multiple objects located at binocular disparities ranging from $-1^{\circ}$ to $-5^{\circ}$, and a single salient object moving from zero disparity to a specific, defined degree of disparity with various 
velocities and toward the viewer, as shown in Figure 3. Note that the binocular disparity $(\eta=\beta-\alpha)$ is the difference between the converging angles of the $3 \mathrm{D}$ object point and the screen.

Table I shows the object's motion-in-depth information in our stereoscopic 3D content. Note that the viewing distance is $D$ and the offset distance is $\Delta D$. We assume that the inter-pupillary distance is $64 \mathrm{~mm}$. Figure 4 shows sample frames from our stereoscopic 3D video along with diverse binocular disparities. The video clips at specific binocular disparity were played at 3-, 5- and 10-minute lengths.

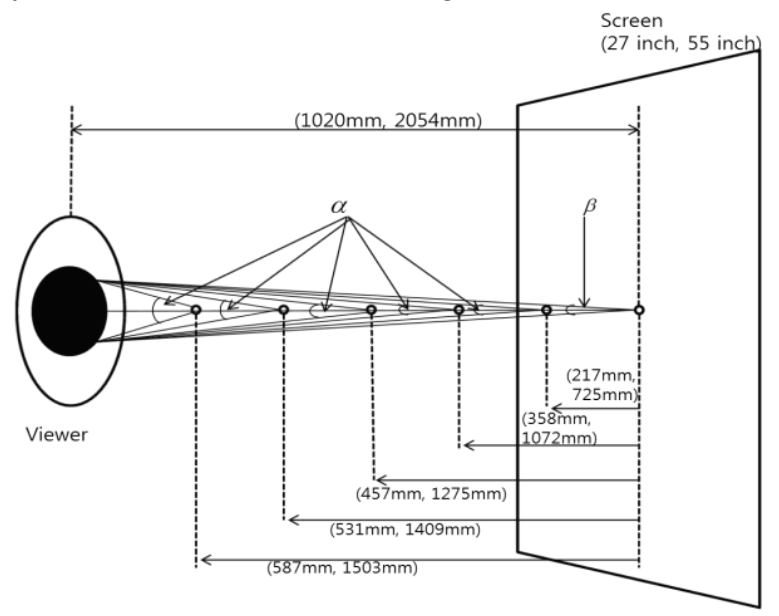

Figure 3: Binocular disparity.

\begin{tabular}{|c|c|c|c|c|c|c|}
\hline Display size & $\begin{array}{c}D \\
(\mathbf{m m})\end{array}$ & $\begin{array}{c}\Delta D \\
(\mathbf{m m})\end{array}$ & $\alpha$ & $\beta$ & $\eta$ & Motion Velocity \\
\hline \hline \multirow{5}{*}{27 inch } & 1020 & 217 & 4.57 & 3.60 & -1 & \\
& 1020 & 358 & 5.54 & 3.60 & -2 & Slow $(105 \mathrm{~mm} / \mathrm{s})$ \\
& 1020 & 457 & 6.51 & 3.60 & -3 & Medium $(200 \mathrm{~mm} / \mathrm{s})$ \\
& 1020 & 531 & 7.50 & 3.60 & -4 & Fast $(257 \mathrm{~mm} / \mathrm{s})$ \\
& 1020 & 587 & 8.47 & 3.60 & -5 & \\
\hline \multirow{5}{*}{55 inch } & 2054 & 725 & 2.76 & 1.78 & -1 & \\
& 2054 & 1072 & 2.73 & 1.78 & -2 & Slow $(105 \mathrm{~mm} / \mathrm{s})$ \\
& 2054 & 1275 & 4.71 & 1.78 & -3 & Medium $(200 \mathrm{~mm} / \mathrm{s})$ \\
& 2054 & 1409 & 5.69 & 1.78 & -4 & Fast $(257 \mathrm{~mm} / \mathrm{s})$ \\
& 2054 & 1503 & 6.66 & 1.78 & -5 & \\
\hline
\end{tabular}

Table 1 : Configuration of our stereoscopic 3D content.

\subsection{Test Procedure}

The test procedure consisted of four stages, as shown in Figure 5. The subject closed their eyes and rested for 5 minutes. This stage was intended to eliminate eyestrain resulting from the subject's previous activities, and to achieve a normalized baseline for the experiment's diverse subjects. Then, the following eight questions were answered in a period of 2 minutes to check the subject's pre-stimulus subjective eyestrain. The questionnaire was similar to those used in previous research $[7,8,10]$. 


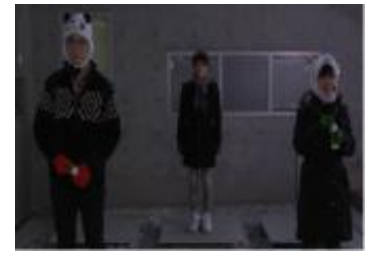

(a) $2 \mathrm{D}$

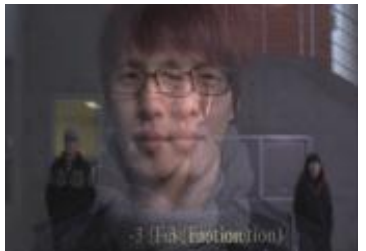

(d) $-3^{\circ}$

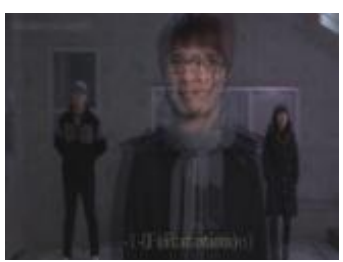

(b) $-1^{\circ}$

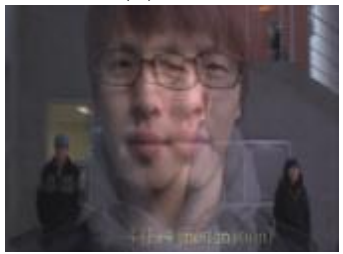

(e) $-4^{\circ}$

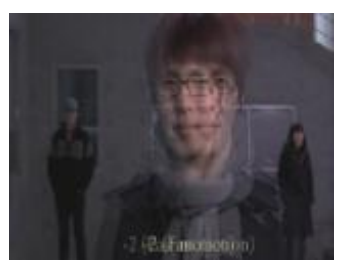

(c) $-2^{\circ}$

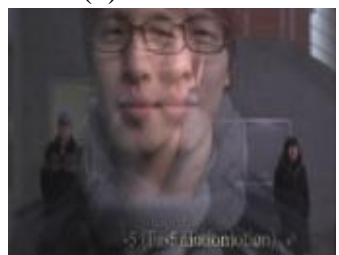

(f) $-5^{\circ}$

Figure 4: Sample frames from our stereoscopic 3D video.

\begin{tabular}{|c|c|c|c|}
\hline Display size & Degree of disparity & Motion Velocity & Viewing time \\
\hline \hline \multirow{2}{*}{27 inch } & 2D & & $3,5,10$ \\
& 3D $\left(-1^{\circ},-2^{\circ},-3^{\circ},-4^{\circ},-5^{\circ}\right)$ & Slow, Medium, Fast & $3,5,10$ \\
\multirow{2}{*}{55 inch } & $2 \mathrm{D}$ & & $3,5,10$ \\
& 3D $\left(-1^{\circ},-2^{\circ},-3^{\circ},-4^{\circ},-5^{\circ}\right)$ & Slow, Medium, Fast & $3,5,10$ \\
\hline
\end{tabular}

Table 2 : Viewing conditions

The questionnaire questions were as follows:

Q1 : My eyes feel tired (eye strain).

Q2 : I feel dizzy looking at the screen.

Q3 : My eyes feel diplopia (double vision).

Q4 : My eyes feel stimulated.

Q5 : I feel blurred vision.

Q6 : My eyes feel dry.

Q7 : I have a headache.

Q8 : I feel lightheaded.

Next, the participant watched the 3-, 5- and 10-minute stereoscopic 3D video clips as shown in Table 2 . As the subject was wearing polarized glasses equipped with an eye tracking device, we detected her eye blinking using eye state diagram as in [16] and measured her eyestrain response at one minute intervals with a hand held slider similar to [2]. The position of the slider could be adjusted along a graphical scale and including at regular intervals the adjective terms, [extremely uncomfortable]-[uncomfortable]-[middle][comfortable]-[very comfortable], in accordance with the ITU recommendation [15]. After watching stereoscopic 3D video, the subject re-answered the previously mentioned eight 
questions in a span of 2 minutes to measure the post-stimulus subjective eyestrain. The survey scores, representing the amount of subjective discomfort, were normalized between 0 and 1 after subtracting the pre-stimulus score from the corresponding post-stimulus score.

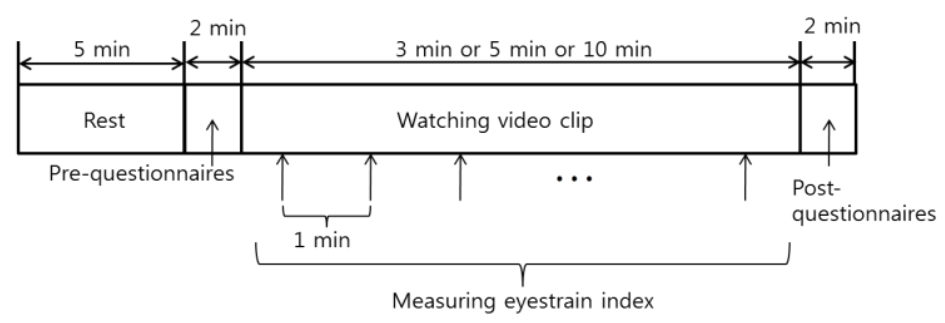

Figure 5: Test procedure.

\section{Experimental Results}

Visual discomfort was measured in respect to three kinds of motion-in-depth (slow, medium and fast motion), viewing time and display size. To begin with, we present our subjective assessment results based on the participants' questionnaires. An analysis of variance (ANOVA) was performed on the individual ratings of discomfort obtained through the questionnaires for each size of display. Note that we only use the median $50 \%$ of rating scores for analysis, while the upper and lower $25 \%$ of rating scores were removed as statistical outliers. As a result, only 6 questions were statistically significant across both sizes of display with a $95 \%$ significance level which is used in [12][16]. With the 27 inch display, the $\mathrm{Q} 1(\mathrm{~F}(5,102)=3.772, \quad \mathrm{p}<0.05), \mathrm{Q} 3(\mathrm{~F}(5,102)=3.127, \mathrm{p}<0.05), \mathrm{Q} 4(\mathrm{~F}(5$, $102)=9.510, \mathrm{p}<0.05), \mathrm{Q} 5(\mathrm{~F}(5,102)=6.658, \mathrm{p}<0.05), \mathrm{Q} 6(\mathrm{~F}(5,102)=10.247, \mathrm{p}<0.05)$, and $\mathrm{Q} 7(\mathrm{~F}(5,102)=2.675, \quad \mathrm{p}<0.05)$ were significant. With the 55 inch display, the $\mathrm{Q} 1(\mathrm{~F}(5$, $102)=4.840, \quad \mathrm{p}<0.05), \mathrm{Q} 3(\mathrm{~F}(5,102)=5.918, \mathrm{p}<0.05), \mathrm{Q} 4(\mathrm{~F}(5,102)=16.130, \quad \mathrm{p}<0.05)$, $\mathrm{Q} 5(\mathrm{~F}(5,102)=14.625, \mathrm{p}<0.05), \mathrm{Q} 6(\mathrm{~F}(5,102)=14.302, \mathrm{p}<0.05)$, and $\mathrm{Q} 7(\mathrm{~F}(5,102)=2.677$, $\mathrm{p}<0.05)$ were significant. All other questions were judged as not significant.

Thus, we set discomfort value as an average of Q1, Q3, Q4, Q5, Q6, and Q7 rating scores. To model the subjective visual discomfort, we fit a two-dimensional function of binocular disparity and viewing time to the data for each motion-in-depth. We use quadratic polynomials fitting method to obtain smooth visual discomfort model because visual discomfort is not abruptly changed on the continuous binocular disparity and viewing time.

Subjective visual discomfort was modeled using the polynomial function $v(d, t)=\rho_{0}+\rho_{1} d+\rho_{2} t+\rho_{3} d^{2}+\rho_{4} d t+\rho_{5} t^{2}$ where $d$ denotes binocular disparity in degree and $t$ is viewing time. Regression coefficients were calculated using a least squares method, which maximizes the Pearson correlation between subjective discomfort and viewing time. Figure 6 and Table 3 show that the subjective discomfort was mainly induced by fast motion-in-depth with respect to binocular disparity and viewing time.

To convert eye blinking rates into objective visual discomfort, we correlate the eye blinking rate with viewers' visual discomfort responses. The relationship between eye blinking rates and visual discomfort is modeled using the polynomial function 
$h(b)=\phi_{0}+\phi_{1} b+\phi_{2} b^{2}$, where $b$ denotes the normalized eye blink rate, as shown in Figure 7 and Table 4.

By integrating eye blinking rates and viewers' discomfort responses, we construct an objective visual discomfort model. For each size of display, the observed eye blink rate corresponds to visual discomfort value by function $h$. From the calculated visual discomfort, we use the polynomial function $u(d, t)=\gamma_{0}+\gamma_{1} d+\gamma_{2} t+\gamma_{3} d^{2}+\gamma_{4} d t+\gamma_{5} t^{2}$ where $d$ denotes binocular disparity and $t$ is the viewing time to obtain the objective visual discomfort model. Regression analysis was performed to find the optimal value of the coefficients. This is shown in Figure 8 and Table 5. Visual discomfort increases rapidly as the degree of binocular disparity increases in case of fast motion-in-depth. In addition, the smaller-sized display results in more eyestrain than the larger-sized display.

\section{Conclusion}

In this paper, we proposed a visual discomfort assessment method for stereoscopic 3D video in respect to depth in motion, viewing time, and display size. We performed an objective assessment test by means of eye blinking rate detection, and a subjective assessment test by means of a questionnaire. By integrating objective and subjective assessment results, we constructed a visual discomfort estimation model. We found that large visual discomfort was experienced by the viewers in case of fast motion-in-depth.

Using our proposed model, it is possible to obtain practical guidelines for visually comfortable stereoscopic 3D content creation. In addition, our experimental data may be applicable to creating safety guidelines for viewing stereoscopic 3D content.

In our future work, we will extend our proposed method for assessing visual discomfort to deal with other factors such as global camera motion and contrast in order to establish safety guidelines in watching stereoscopic 3D video.

\begin{tabular}{|c|c|c|c|c|c|c|c|}
\hline Display size & Velocity & $\rho_{0}$ & $\rho_{1}$ & $\rho_{2}$ & $\rho_{3}$ & $\rho_{4}$ & $\rho_{5}$ \\
\hline \hline \multirow{3}{*}{27} & $\mathrm{~S}$ & -0.0113 & 0.0039 & 0.0178 & 0.0022 & -0.006 & -0.0016 \\
& $\mathrm{M}$ & -0.0196 & -0.0058 & 0.0247 & 0.0006 & -0.0052 & -0.0019 \\
& $\mathrm{~F}$ & -0.0168 & 0.0079 & 0.0291 & 0.0039 & -0.0068 & -0.0025 \\
& $\mathrm{~S}$ & -0.0183 & -0.0007 & 0.0172 & 0.0018 & -0.0046 & -0.0015 \\
55 & $\mathrm{M}$ & -0.0219 & -0.0146 & 0.0195 & -0.0016 & -0.0054 & -0.0016 \\
& $\mathrm{~F}$ & -0.0124 & 0.0047 & 0.0217 & 0.0026 & -0.006 & -0.0018 \\
\hline
\end{tabular}

Table 3: Regression coefficients of subjective discomfort in terms of binocular disparity and viewing time(with $95 \%$ confidence bounds). 

MOTION-IN-DEPTH IN STEREOSCOPIC 3D VIDEO
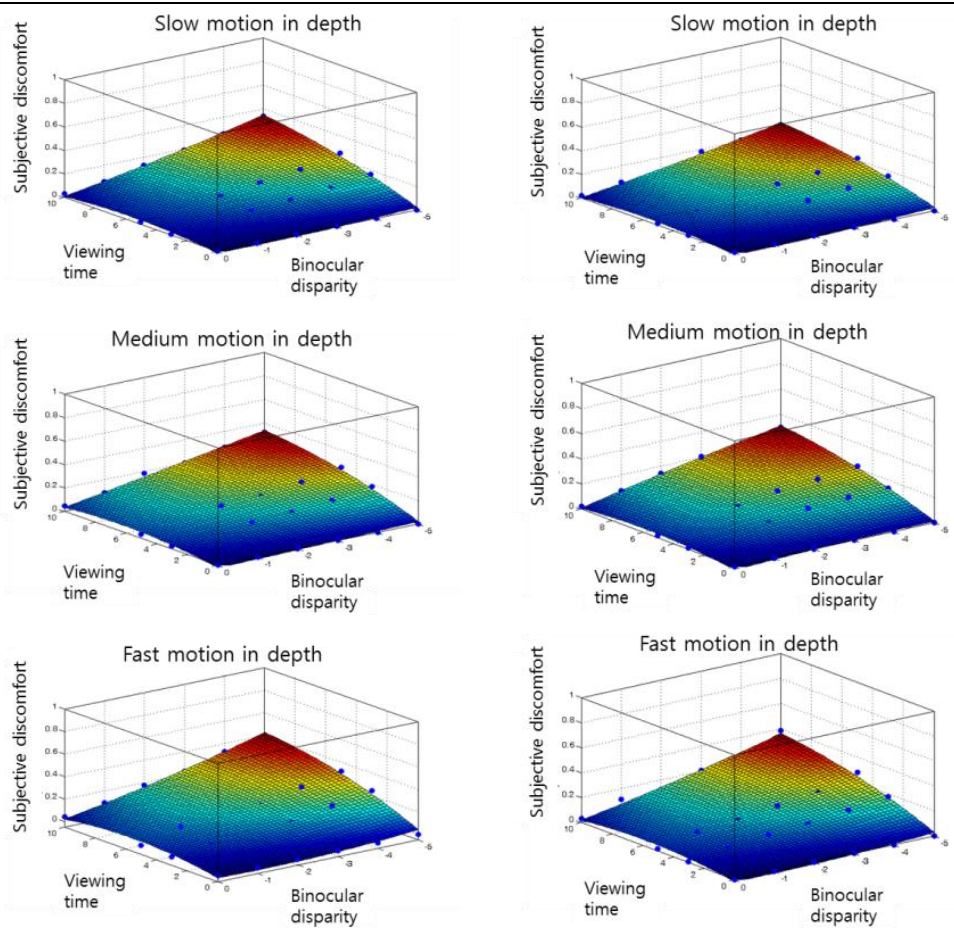

(a)

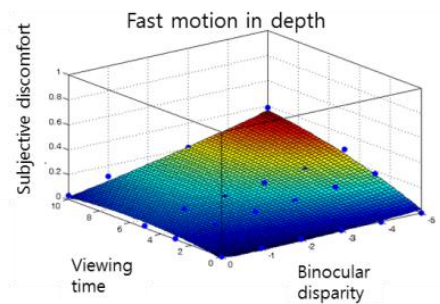

(b)

Figure 6: Subjective visual discomfort : (a) 27 inch display; (b) 55 inch display.

\begin{tabular}{|c|c|c|c|c|}
\hline Display size & $\phi_{0}$ & $\phi_{1}$ & $\phi_{2}$ & Pearson's coefficient \\
\hline \hline 27 & -0.1948 & 1.2120 & 0.0137 & 0.8745 \\
55 & -0.2585 & 2.0300 & -0.6838 & 0.8359 \\
\hline
\end{tabular}

Table 4: Regression coefficients between eye blinking rates and visual discomfort(with $95 \%$ confidence bounds)

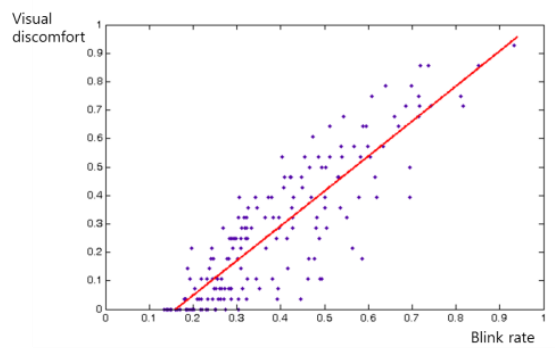

(a)

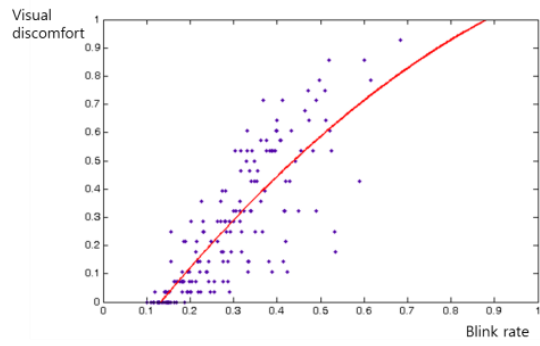

(b)

Figure 7: Relationship between visual discomfort and eye blinking rates : (a) 27 inch; (b) 55 inch 


\begin{tabular}{|c|c|c|c|c|c|c|c|}
\hline Display size & Velocity & $\gamma_{0}$ & $\gamma_{1}$ & $\gamma_{2}$ & $\gamma_{3}$ & $\gamma_{4}$ & $\gamma_{5}$ \\
\hline \hline \multirow{3}{*}{27} & $\mathrm{~S}$ & -0.0314 & -0.0043 & 0.0016 & 0.0081 & -0.0053 & 0.0008 \\
& $\mathrm{M}$ & -0.0089 & -0.0238 & 0.0116 & 0.0085 & -0.0066 & -0.0003 \\
& $\mathrm{~F}$ & 0.1276 & -0.0107 & -0.0182 & 0.0121 & -0.0082 & 0.0024 \\
& $\mathrm{~S}$ & -0.0782 & -0.0762 & 0.0008 & -0.0017 & -0.0031 & 0.0002 \\
55 & $\mathrm{M}$ & -0.0548 & -0.0714 & 0.0008 & 0.0025 & -0.0026 & 0.0005 \\
& $\mathrm{~F}$ & -0.0137 & -0.07 & 0.0078 & 0.0093 & -0.0005 & 0.0008 \\
\hline
\end{tabular}

Table 5: Regression coefficients of objective visual discomfort in terms of binocular disparity and viewing time(with $95 \%$ confidence bounds)
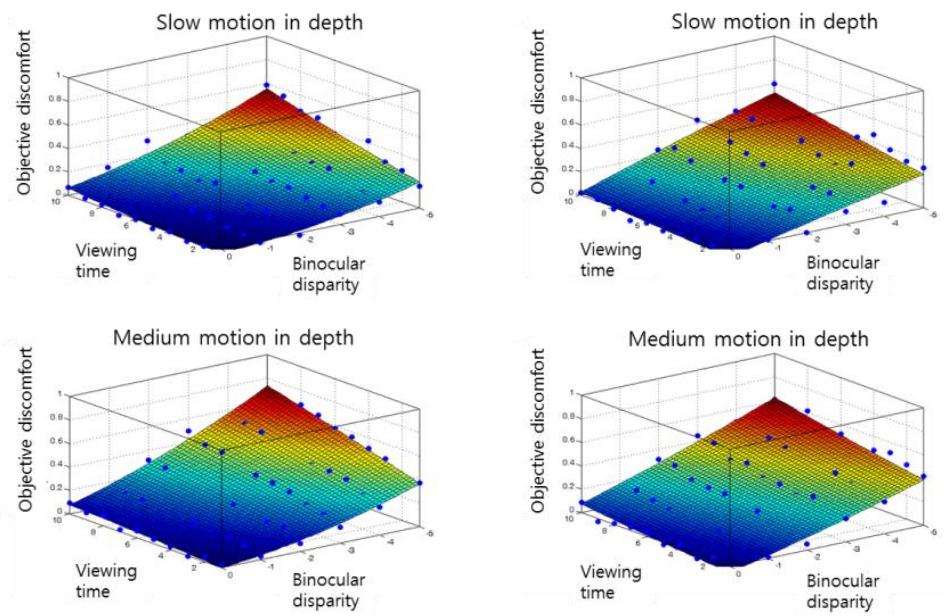

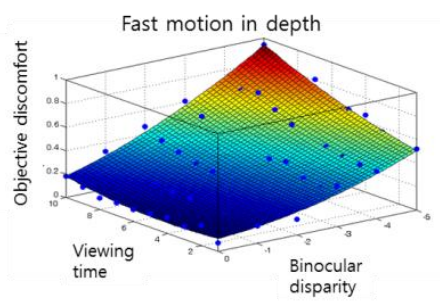

(a)

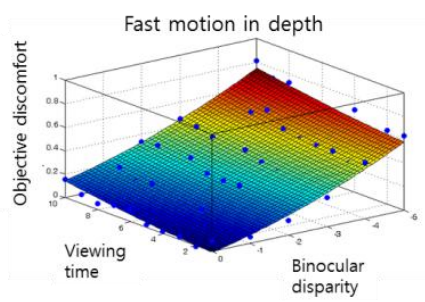

(b)

Figure 8: Objective visual discomfort : (a) 27 inch; (b) 55 inch

\section{Acknowledgements}

This work was supported by the Industrial Strategic technology development program(10041937, Development of Personalized Stereoscopic 3D Editing Tool and Rendering Process) funded by the Ministry of Knowledge Economy(MKE, Korea) 


\section{References}

[1] M. Lambooij, W. IJsselsteijn, M. Fortuin, and I. Heynderickx, "Visual discomfort and visual fatigue of stereoscopic displays: A review," J.Imaging Sci. Technol., vol. 53, no. 3, pp. 030201030201-14, 2009.

[2] M. Lambooij, W.A. IJsselsteijn, I. Heynderickx, "Visual discomfort of 3D TV: Assessment methods and modeling," Displays, vol. 32, Issue 4, Oct. 2011.

[3] D. M. Hoffman, A. R. Girshick, K. Akeley, and M. S. Banks, "Vergence-accommodation conflicts hinder visual performance and cause visual fatigue", Journal of Vision, vol. 8, pp.1-30, 2008 .

[4] N. Hiruma and T. Fukuda, "Accommodation response to binocular stereoscopic TV images and their viewing conditions,” SMPTE J., vol. 102, no. 12, pp. 1137-1144, 1993.

[5] Y. Okada, K. Ukai, J. S. Wolffsohn, B. Gilmartin, A. Iijima, and T. Bando,"Target spatial frequency determines the response to conflicting defocusand convergence-driven accommodative stimuli”, Vision Res, vol. 46, pp. 475-484, 2006.

[6] T. Inoue and H. Ohzu, "Accommodative responses to stereoscopic three-dimensional display", Appl. Opt, vol. 36, pp. 4509-4515, 1997.

[7] S. Yano, S. Ide, T. Mitsuhashi, and H. Thwaites, "A study of visual fatigue and visual comfort for 3D HDTV/HDTV images,” Displays, vol. 23, pp. 191-201, 2002.

[8] F. Speranza, W. J. Tam, R. Renaud, and N. Hur, "Effect of disparity and motion on visual comfort of stereoscopic images," in Proc. of SPIE, vol 6055, pp. 94-103, 2006.

[9] Y.J. Jung, S. Lee, H. Sohn, H. W. Park and Y. M. Ro, "Visual comfort assessment metric based on salient object motion information in stereoscopic video," Journal of Electron Imaging, vol. 21, Issue 1, Feb, 2012.

[10] F. L. Kooi and A. Toet, "Visual comfort of binocular and 3D displays," Displays, vol. 25, pp. 99-108, 2004.

[11] M. Wopking, "Viewing comfort with stereoscopic pictures: An experimental study on the subjective effects of disparity magnitude and depth of focus," J. SID, vol. 3, no. 3, pp. 101-103, 1995.

[12] L. E. Coria, Xu Di and P. Nasiopoulos, "Quality of experience of stereoscopic content on displays of different sizes: A comprehensive subjective evaluation," IEEE International Conference on Consumer Electronics (ICCE) , pp.755-756, Jan, 2011.

[13] L.R. Harris and M. Jenkin, Vision in 3D Environments, Cambridge University Press, 2011.

[14] S. Yano, M. Emoto and T. Mitsuhashi, "Two factors in visual fatigue caused by stereoscopic HDTV images," Displays, vol. 25, no. 4, pp.141-150, 2004.

[15] ITU, Methodology for the subjective assessment of the quality of television pictures, Recommendation BT.500-13 2010.

[16] S. -H Cho and H. -B. Kang, "The measurement of eyestraincaused from diverse binocular disparities, viewing time and display sizes inwatching stereoscopic 3D content," Proc. IEEE 3DCINE Workshop in conjunction with CVPR 2012, pp.23-28, June, 2012. 